\title{
Wideband Underwater Acoustic CDMA: Adaptive Multichannel Receiver Design
}

\author{
Milica Stojanovic \\ Massachusetts Institute of Technology \\ Cambridge, MA 02139 \\ millitsa@mit.edu
}

\author{
Lee Freitag \\ Woods Hole Oceanographic Institution \\ Woods Hole, MA 02543 \\ lfreitag@whoi.edu
}

\begin{abstract}
Two receivers that utilize multichannel (array) processing of asynchronous wideband direct-sequence CDMA signals are proposed: the symbol decision feedback (SDF) receiver and the chip hypothesis feedback (CHF) receiver. In the SDF configuration, receiver parameters are adapted at the symbol rate. This limits its applicability to the channels whose time variation is slow compared to the symbol rate, which may not be the case in a mobile underwater acoustic system. In the CHF configuration, the receiver parameters are adapted at the chip rate, and decision-feedback equalization is accomplished at chip resolution. At the price of increased computational complexity (linearly proportional to the modulation level) this receiver provides improved performance for systems where time variation cannot be neglected with respect to the symbol rate. Performance of the two receivers was demonstrated in a fouruser scenario, using experimental data obtained over a $2 \mathrm{~km}$ shallow water channel. At the chip rate of 19.2 kilochips per second with QPSK modulation, excellent results were achieved at an aggregate data rate of up to $10 \mathrm{kbps}$.
\end{abstract}

\section{INTRODUCTION}

Code-division multiple-access (CDMA) based on directsequence (DS) spread-spectrum modulation is considered for the future mobile underwater wireless communication networks [1]. In addition to inherent multipath diversity, DS spread-spectrum provides a natural platform for operation in a hostile environment. At this time, it is practical to conceive of a network scenario in which a base station serves several mobile users such as autonomous underwater vehicles (AUVs) within a radius of few kilometers. This paper deals with the design of communication methods suitable for multichannel (array) detection of signals in such a network.

The major obstacles encountered on an underwater channel are extended multipath propagation and rapid time variation, which cause severe frequency-selective fading in a wideband acoustic system. Due to the limited bandwidth, which may be on the order of $10 \mathrm{kHz}$ for a system operating over few kilometers, a system is designed for transmission at maximal chip rate, while the achievable bit rate is determined by the spreading factor, or the processing gain. The spreading factor is selected to support the desired number of users and provide the necessary quality of performance. At the same time, it is desired that as high a bit rate as possible be achieved. These requirements dictate the choice of the spreading factor on the order of few tens. A higher processing gain is needed in systems that seek to provide low probability of intercept (LPI) in addition to supporting multiple users.

Because of the severe frequency-selective distortion caused by multipath propagation, it is beneficial, if not imperative, to use a decision-feedback equalizer over underwater acoustic channels of interest. In particular, multichannel decisionfeedback equalization is considered to be the most effective method for detection of non-spread, bandwidth efficient signals in underwater acoustic channels [2]-[4].

Multipath interference, seen as intersymbol interference (ISI) in a non-spread system, appears as interchip interference in a DS spread-spectrum system. In a typical shallow water channel, the multipath spread may be on the order of $10 \mathrm{~ms}$, resulting in multipath interference that can span a hundred chip intervals. Thus, not only is the interchip interference severe, but the intersymbol interference cannot be neglected. In contrast to this situation, ISI in most of the operating DS CDMA radio systems can be neglected [5]. However, as wideband radio systems come into use, ISI emerges as a problem in these systems as well. Various approaches to combining equalization with multiple-access interference suppression have been proposed for these systems. In particular, references [6]-[9] address the methods for decisionfeedback equalization. In all of these receivers, adaptation of the equalizers is performed at the symbol rate, which limits their applicability to those channels that do not change rapidly at the symbol level. Another design, proposed in [10] for rapidly varying underwater acoustic channels, is based on chip-rate adaptive equalization, which is made possible by feeding back hypothesized chip values. At the price of increased computational complexity, this receiver gains the ability to perform chip-resolution feedback filtering and fast, chip-rate adaptation.

In this paper, two types of minimum mean squared error (MMSE) decision-feedback receivers are proposed for use in a multichannel configuration. The first receiver is based on the principle of symbol decision feedback (SDF), while the second receiver is based on the principle of chip hypothesis feedback (CHF). A multichannel combiner [3] and a phase synchronization method are integrated into the receivers to enable their operation in realistic mobile underwater acoustic systems.

Performance of these receivers is demonstrated using ex- 
perimental data. A four-user CDMA system is considered, operating at 19.2 kilochips per second (kcps) using QPSK modulation and varying spreading factors, over a $2 \mathrm{~km}$ shallow water channel. Excellent results achieved through multichannel processing demonstrate the possibility to support multiple users in a highly distorted underwater channel, even at minimal processing complexity and low bandwidth expansion.

The paper is organized as follows. In Sec.II, receiver algorithms are presented. Experimental results are discussed in Sec.III. Sec.IV summarizes the conclusions.

\section{RECEIVER Algorithm}

\section{A. System model}

The complex baseband transmitted signal of the $i$ th user is given by

$$
u_{i}(t)=\sum_{n} d_{i}(n) g_{i}(t-n T), \quad i=1, \ldots I
$$

where $d_{i}(n)$ represents the data sequence, $T$ is the symbol period, and $g_{i}(t)$ is the spreading waveform. The data symbols can take values from an arbitrary QAM or PSK alphabet. The spreading waveform is given by

$$
g_{i}(t)=\sum_{l=0}^{L-1} p_{i}(l) g\left(t-l T_{c}\right)
$$

where $p_{i}(l), l=0, \ldots L-1$, is the chip sequence of the $i$ th spreading code, $T_{c}$ is the chip interval, and $g(t)$ is the transmitter shaping pulse. The code period is equal to the symbol duration, $L T_{c}=T$. The chip sequence can be complex, or real-valued $( \pm 1)$. The transmitted signal can alternatively be expressed as

$$
u_{i}(t)=\sum_{k} q_{i}(k) g\left(t-k T_{c}\right)
$$

where $q_{i}(k)$ is the modulated chip sequence,

$$
q_{i}(n L+l)=d_{i}(n) p_{i}(l), \quad l=0, \ldots L-1, \forall n
$$

The signals of different users travel through different, multipath-distorted channels, and arrive at a common receiver. Below, we describe two receivers, one based on symbol decision feedback, and another based on chip hypothesis feedback.

\section{B. Symbol decision feedback (SDF) receiver}

The block diagram of the SDF receiver is shown in Fig.1. The same receiver structure is used for each desired user, whose index $i$ we drop for simplicity of notation, i.e. $d(n)=$ $d_{i}(n)$ and $p(l)=p_{i}(l)$ in assumed what follows. The received complex baseband signal observed at the $k$ th array element is denoted by $v_{k}(t), k=1, \ldots K$. The signals are sampled at the Nyquist or higher rate, resulting in $N_{s}$ samples per chip interval $T_{c}$. The $K$ received signals are first spatially combined to form $P \leq K$ inputs for the feedforward filters. The combiner coefficients are arranged into $K$-element column vectors $\mathbf{c}_{p}, p=1, \ldots P$. The feedforward filters are fractionally spaced at $T_{c} / N_{s}$, and each has $N$ coefficients arranged in a column vector $\mathbf{a}_{p}, p=1, \ldots P$. The temporal samples of the $k$ th receive element are arranged into an $N$ element vector $\mathbf{v}_{k}(n, l)=\mathbf{v}_{k}(n L+l)=\left[\ldots v_{k}\left(n T+l T_{c}+\right.\right.$ $\left.\left.T_{s}\right) v_{k}\left(n T+l T_{c}\right) v_{k}\left(n T+l T_{c}-T_{s}\right) \ldots\right]^{T}$, where the time index $(n, l)$ refers to the $l$ th chip of the $n$th data symbols. It is assumed that coarse time-synchronization is performed such that the vectors $\mathbf{v}_{k}(n, l), l=0, \ldots L-1$ contain significant contribution of the $n$th transmitted data symbol $d(n)$. The signal vectors of all receive elements form the signal matrix

$$
\mathbf{V}(n, l)=\mathbf{V}(n L+l)=\left[\begin{array}{c}
\mathbf{v}_{1}^{T}(n, l) \\
\vdots \\
\mathbf{v}_{K}^{T}(n, l)
\end{array}\right], l=0, \ldots L-1
$$

The signals at the output of the feedforward equalizers are summed and synchronized using a phase estimate $\hat{\theta}(n)$. In the majority of applications, it is sufficient to use a single carrier phase estimate, and we do so for the sake of computational efficiency. Extension to multiple carrier estimates (one per input channel, or one per equalizer input) is straightforward. The feedback filter has $M$ coefficients arranged into a vector b. During the detection of the $n$th data symbol, the feedback filter operates on previous symbols, $\tilde{\mathbf{d}}(n-1)=[\tilde{d}(n-$ 1) $\ldots \tilde{d}(n-M)]^{T}$, which represent the known data symbols during training, and decisions thereafter. The estimate of the $n$th data symbol is obtained as

$$
\hat{d}(n)=\frac{1}{L} \sum_{l=0}^{L-1} p^{*}(l) \sum_{p=1}^{P} \mathbf{c}_{p}^{\prime} \mathbf{V}(n, l) \mathbf{a}_{p}^{*} e^{-j \hat{\theta}(n)}-\mathbf{b}^{\prime} \tilde{\mathbf{d}}(n-1)
$$

where prime denotes conjugate transpose. This expression defines the dependence of the data estimate on all the receiver parameters - the combiner weights, the feedforward filters, the feedback filter, and the phase estimate. These parameters are jointly adjusted to minimize the MSE in data detection. The adaptive algorithm is driven by a single error, $e(n)=$ $\tilde{d}(n)-\hat{d}(n)$.

Adaptation of the SDF receiver parameters is performed at the symbol rate. As symbol decisions become available at the end of the despreading period, they are fed back to the equalizer, and also used to generate the error that drives the adaptive algorithm. The receiver parameters are thus updated every $L$ chip intervals. At a fixed chip rate (normally chosen maximal within the available acoustic bandwidth) this means that by increasing the spreading factor the symbol duration increases, thus allowing for a greater change to occur in the channel between successive updates. Hence, while the interference suppression capability improves with increased spreading factor, the channel tracking capability degrades. In conditions of rapid channel variation, which may arise in mobile acoustic channels, this fact may lead to a counter-productive effect whereby increasing the spreading factor results in performance degradation.

The Doppler frequency, given by $f_{d}=f_{c} v / c$ for a carrier frequency $f_{c}$ and a vehicle speed $v$, can become quite large as compared to the symbol rate because of the low speed of sound (nominal value is $c=1500 \mathrm{~m} / \mathrm{s}$ ). For example, at $f_{c}=33 \mathrm{kHz}$, and a chip rate of $R_{c}=19.2 \mathrm{kcps}$, which are the values used for 


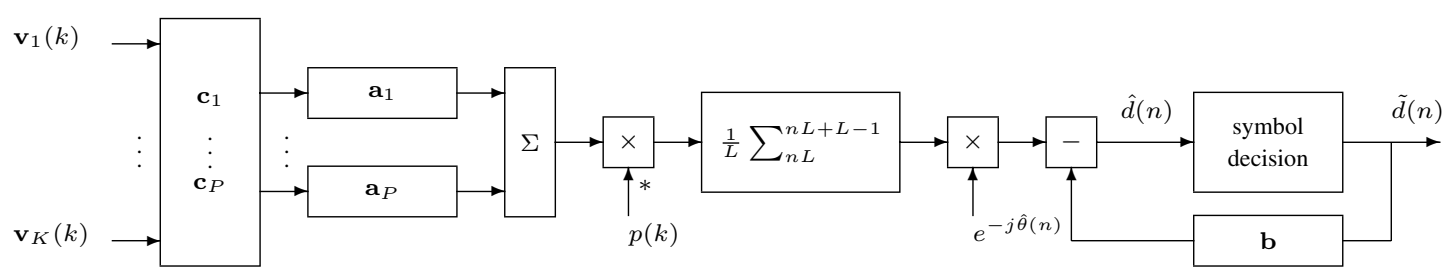

Fig. 1. DS receiver based on multichannel (many-to-few) combining and symbol decision feedback (SDF) adaptive equalization. Symbol decisions are fed back and used to update the combiner, the equalizer filters, and the phase estimate. The feedforward filters have fractionally chip-spaced taps. The feedback filter has symbol-spaced taps. Receiver parameters are updated at the symbol rate. Adaptive algorithm is driven by the symbol error, $\tilde{d}(n)-\hat{d}(n)$.

the experimental system, the normalized Doppler frequency is $f_{d} T=0.001 v L$. The velocity of an AUV is on the order of few $\mathrm{m} / \mathrm{s}$, and, hence, the factor $f_{d} T$ can become large even at moderate spreading factor $L$. The channel variation over one symbol interval then becomes too large to be tracked by an adaptive algorithm, causing the performance of the SDF receiver to deteriorate, or eventually to fail. This fact serves as a motivation to investigate a different type of adaptive receiver, in which parameter updates are performed at the chip rate rather than the symbol rate.

\section{Chip hypothesis feedback (CHF) receiver}

The block diagram of the CHF receiver is shown in Fig.2. The feedback filter is chip-spaced, and all the receiver parameters are updated at the chip rate. Despreading and symbol decision are performed separately, using the estimated chip sequence. However, in order to enable chip-rate filtering and adaptation, chip decisions must be available before despreading has been completed and symbol decision made. Hence, the symbol decision is not available at the time when it is needed to generate the current chip decision. To overcome this problem, a set of hypotheses is generated for each symbol interval. The principle of hypothesis feedback equalization is given in [10] for the single channel receiver configuration. This principle remains the same for the multichannel configuration, and we only briefly summarize its key points.

Each hypothesis corresponds to one possible value of the transmitted data symbol. For QPSK, $H=4$ hypotheses are made at the beginning of each symbol interval, denoted by superscript $h$ for the desired user $i: d_{i}^{h}(n)=e^{j(2 h-1) \pi / 4}, h=$ $1, \ldots 4$. Under each hypothesis $h$, the modulated chip sequence is known for the $L$ chips of the $n$th symbol interval:

$$
q_{i}^{h}(n L+l)=d_{i}^{h}(n) p_{i}(l), \quad l=0, \ldots, L-1
$$

Using the resulting four sequences, four adaptive algorithms ( $H$ in general) are run in parallel for $L$ iterations. The output of the algorithm corresponding to hypothesis $h$ is the sequence of chip estimates $\hat{q}_{i}^{h}(n L+l), l=0, \ldots, L-1$. These estimates are used by the despreader to form the symbol estimates

$$
\hat{d}_{i}^{h}(n)=\frac{1}{L} \sum_{l=0}^{L-1} \hat{q}_{i}^{h}(n L+l) p_{i}^{*}(l), \quad h=1, \ldots H
$$

The data estimate of the correct hypothesis should closely resemble the hypothesized symbol value. Thus, the squared error

$$
E_{i}^{h}(n)=\left|d_{i}^{h}(n)-\hat{d}_{i}^{h}(n)\right|^{2}
$$

can be used to select the best hypothesis:

$$
\tilde{h}=\arg \min _{h=1, \ldots H} E_{i}^{h}(n)
$$

The corresponding symbol decision is

$$
\tilde{d}_{i}(n)=d_{i}^{\tilde{h}}(n)
$$

At the end of each symbol interval, when the best hypothesis is selected, the combiner coefficients, the equalizer coefficients (together with the feedback filter content) and the phase estimate corresponding to the winning hypothesis are used as initial values for the new symbol interval.

Dropping the desired user's index $i$, the chip estimate at the output of the front section is expressed as

$$
\hat{q}^{h}(k)=\sum_{p=1}^{P} \mathbf{c}_{p}^{h \prime} \mathbf{V}(k) \mathbf{a}_{p}^{h *} e^{-j \hat{\theta}^{h}(k)}-\mathbf{b}^{h \prime} \tilde{\mathbf{q}}^{h}(k-1)
$$

The combiner, the equalizer and the phase estimate corresponding to hypothesis $h$ are labeled accordingly. In this receiver, the phase estimate is obtained every chip interval, and the feedback filter operates on the previous chips, $\tilde{\mathbf{q}}^{h}(k-1)=$ $\left[\tilde{q}^{h}(k-1) \ldots \tilde{q}^{h}(k-M)\right]^{T}$. Note that the feedback filter length $M$ should now be chosen in accordance with the multipath spread measured in chips, not symbols. The chips stored at time $k=n L+l$ in the feedback filter contain $l$ hypothesized values as the most recent entries, while the remaining $M-l$ values correspond to the spreading code modulated by the previous symbol decisions $\tilde{d}(n-1), \tilde{d}(n-2)$, etc.

Optimization of the CHF receiver is based on the MMSE criterion. Adaptation of all the receiver parameters is now driven by the chip estimation error, $e_{q}^{h}(k)=\tilde{q}^{h}(k)-\hat{q}^{h}(k)$. Expression (12) shows the dependence of the chip estimate on all the receiver parameters, and serves as a basis for deriving the adaptive MMSE algorithm. Details of algorithm derivation and adaptive implementation for both the SDF and the CHF receiver can be found in [11]. 


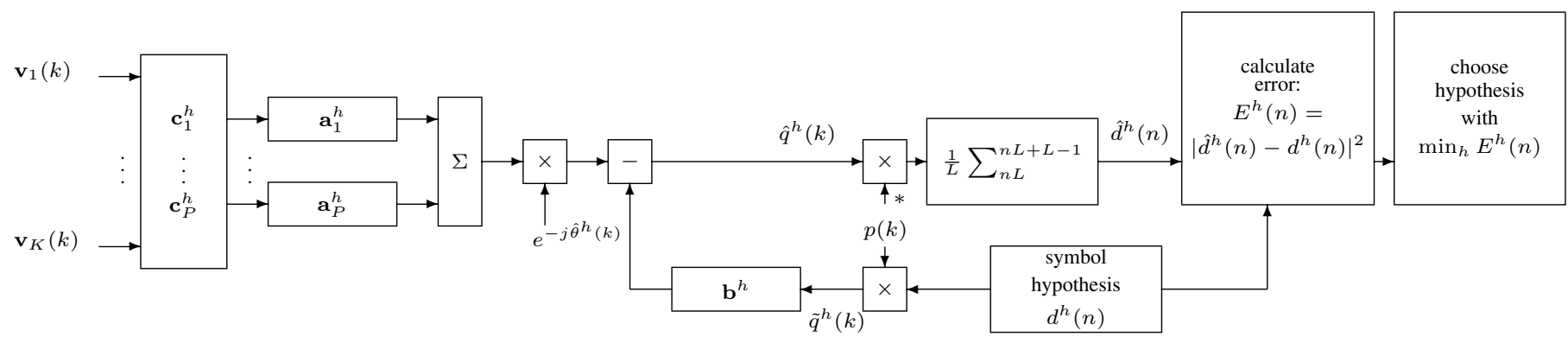

Fig. 2. DS receiver based on multichannel (many-to-few) combining and chip hypothesis feedback (CHF) adaptive equalization. One symbol worth of chip hypotheses are fed back and used to update the combiner, the equalizer filters, and the phase estimate. The feedforward filters have fractionally chip-spaced taps. The feedback filter has chip-spaced taps. Receiver parameters are updated at the chip rate. Adaptive algorithm is driven by the chip error, $\tilde{q}^{h}(k)-\hat{q}^{h}(k)$.

The implementation complexity of the CHF receiver is at least four times that of the SDF receiver for a quaternary modulation method (twice for binary). This price is paid to recover the performance on a rapidly varying channel.

\section{EXPERIMENTAL RESULTS}

\section{A. Signal design and the experiment}

Direct sequence CDMA signals were designed for four users. The modulation method was QPSK, and the same binary sequence was used for spreading the in-phase and quadrature components. Kasami spreading codes of length 15 , 63 and 255 were used (there are 4,8 and 16 codes of these lengths, respectively). At the chip rate of $R_{c}=19.2 \mathrm{kcps}$, the corresponding bit rates were $2,500 \mathrm{bps}, 600 \mathrm{bps}$ and $150 \mathrm{bps}$ per user, resulting in an aggregate bit rate of $10 \mathrm{kbps}, 2.4$ kbps and $0.6 \mathrm{kbps}$, respectively. Pulse shaping was performed using square-root raised-cosine pulses with roll-off factor 0.25 , and the signals were modulated using the carrier frequency $f_{c}=33 \mathrm{kHz}$. Transmission was organized in packets of equal duration. A channel probe consisting of a $100 \mathrm{~ms}$ frequency sweep spanning the bandwidth between $21 \mathrm{kHz}$ and $45 \mathrm{kHz}$ was transmitted between the packets.

The experiment took place the fall of 2003, near the Elba island in Italy. The range between the transmitter and receiver was $2.3 \mathrm{~km}$, in water depth of about $100 \mathrm{~m}$. The signals were received over a 12-element array, submerged at $30 \mathrm{~m}$, with elements equally spaced by $0.15 \mathrm{~m}$.

All the signal processing was performed in baseband, after demodulation using nominal carrier and downsampling to $N_{s}=2$ samples per chip. The multiuser test signals were constructed by adding a desired number of individual users' signals.

\section{B. Channel characteristics}

Figure 3 shows the magnitudes of the channel responses obtained by matched filtering to the frequency sweep probe. Each column contains six responses observed at six of the array elements (every other). The first column represents the time instant prior to transmission of the $L=15$ group of four users' signals; the second and third columns correspond to $L=63$ and 255, respectively. Hence, shown vertically is the spatial variation of the channel response, while the horizontal sequence of responses shows their variation in time. Clearly, both types of variation are present in the channel. The channel exhibits strong multipath and a moderate delay spread of several milliseconds. This delay spread is on the order of few tens of chip intervals in the wideband system considered. In most of the cases, there are two pronounced multipath components, separated by approximately 12.5 chips. These components are followed by distant echoes of decaying energy.
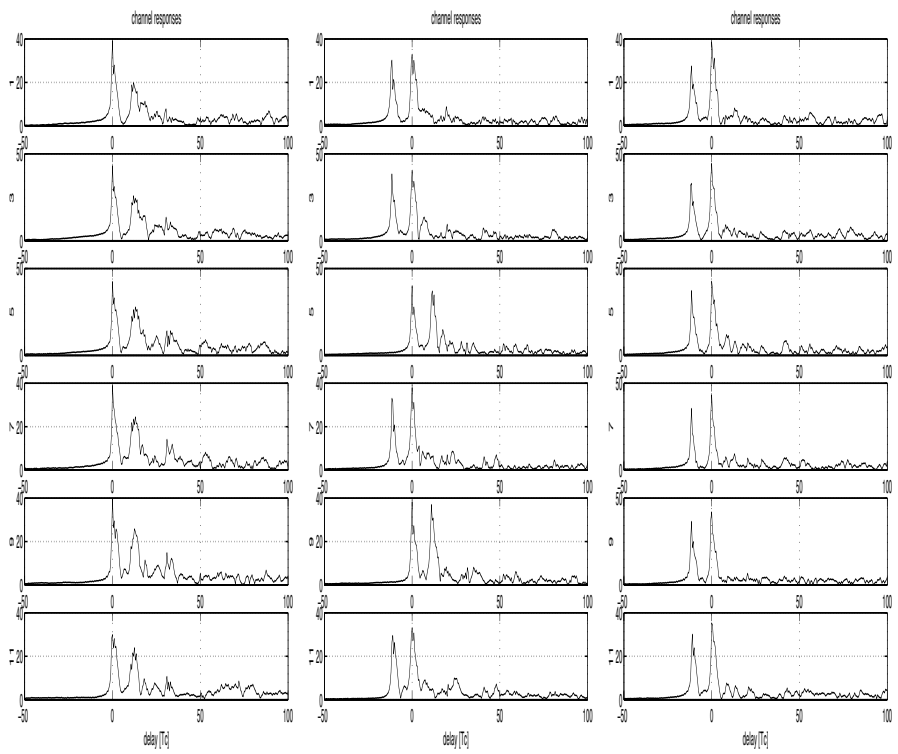

Fig. 3. Channel responses are obtained by matched filtering to the frequency sweep channel probe. Each column contains the responses across the array, observed at a vertical spacing of $0.3 \mathrm{~m}$. The three columns correspond to three instants in time, separated by 1.5 minutes, at which the $L=15,63$ and 255 groups of signals are transmitted.

Channel responses observed during transmission of the $L=63$ group of signals (middle column) show an interesting behavior. The two principal arrivals vary in strength as the depth changes, such that the early arrival is perceived as strongest at some array elements, while the late arrival appears as strongest at other elements. A similar observation can be made by looking at the channel response in time. The time variation of the channel causes the early arrival to appear as strongest at some times, yielding to the late arrival at 
other times. Clearly, the channel is rapidly varying even in the absence of intentional motion, causing the multipath interference to appear causal or anti-causal at different points in time and space. A mode detailed analysis [11] reveals that the coherence time of this channel is on the order of a second. This value implies a normalized Doppler spread on the order of $10^{-5} \mathrm{~L}$, which is well within the tracking capabilities of chiprate adaptive algorithms considered, but approaches the limit of symbol-rate adaptation capability as the spreading factor increases to more than a hundred.

\section{Performance results}

The SDF and the CHF receiver algorithms were applied to the complex baseband received signals. A multiuser test signal was constructed by adding the signals of all four users. The signals were added at equal power and asynchronously. The desired user is arbitrarily chosen as user 1, while the interfering users' signals arrive at relative delays of 8,4 , and 2 chip intervals.

The sizes of equalizer filters are determined from the estimated channel responses so as to span the significant delay spread of the channel currently observed. The SDF receiver used an RLS combiner and an LMS equalizer. The CHF receiver used the LMS algorithm for both the combiner and the equalizer. This choice was made to offset the complexity of hypothesis feedback detection, where four adaptations are carried out in parallel. The SDF receiver benefits greatly from the RLS combiner which provides fast convergence. The LMS step sizes and the RLS forgetting factors were determined experimentally and in accordance with the number of receiver coefficients. The proportional tracking constant $K_{f 1}$ of the second-order phase-locked loop (PLL) was set to 0.0005 for the chip-rate filtering, and scaled by the spreading factor $L$ for symbol-rate processing. The PLL integral tracking constant was set to $K_{f 2}=K_{f 1} / 10$. Various number of array elements $K$ and equalizer branches $P$ were tested. Here, we report on a set of results that utilized all 12 elements of the array.

Figure 4 illustrates the performance of the $\mathrm{CHF}$ receiver at the spreading factor $L=15$. Shown in the figure are the measured mean squared error, the phase estimate, and the output scatter plot. Receiver parameters are listed in the figure, together with the number of training symbols, $N_{t}$. Listed in the figure are also the Doppler frequency $f_{d}$ measured from the phase estimate, the ratio of erroneously detected symbols $P_{e}$, and the output SNR measured from the estimated data symbols. At $L=15$, the performance of the SDF and the $\mathrm{CHF}$ receivers is similar. Very good results obtained at this lowest value of spreading factor owe to the use of multichannel combining. With a single array element, detection fails completely at $L=15$. At least 6 array elements were needed to ensure convergence. When all $K=12$ elements are used, $P=4$ short equalizers provide very good performance. Each feedforward filter captures the principal arrival only using $N=6$ taps, while the feedback length of $M=2$ symbols in the SDF receiver or 30 chips in the $\mathrm{CHF}$ receiver suffices to span the channel response. An alternative design with $P=2$ and $N=28$ provided similar performance. This result serves as a major encouragement as it demonstrates the possibility to support four users with minimal bandwidth expansion using a receiver of extremely low computational complexity.
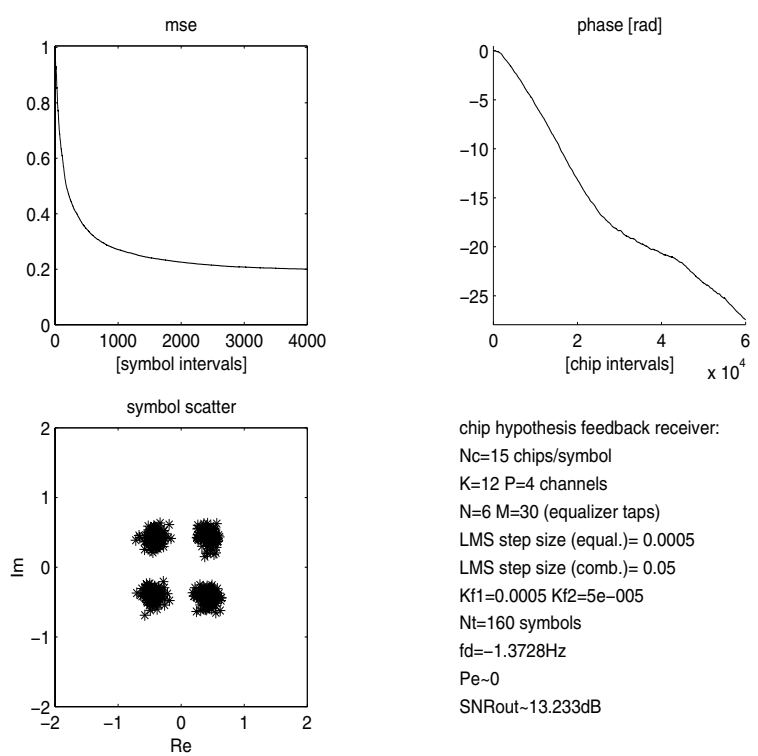

chip hypothesis feedback receiver: $\mathrm{Nc}=15$ chips/symbol $\mathrm{K}=12 \mathrm{P}=4$ channels $\mathrm{N}=6 \mathrm{M}=30$ (equalizer taps) LMS step size (equal.) $=0.0005$ LMS step size (comb.) $=0.05$ $\mathrm{Kf} 1=0.0005 \mathrm{~K} \mathfrak{2} 2=5 \mathrm{e}-005$ $\mathrm{Nt}=160$ symbols $\mathrm{fd}=-1.3728 \mathrm{~Hz}$ $\mathrm{Pe} \sim 0$ SNRout $13.233 \mathrm{~dB}$

Fig. 4. Performance of the $\mathrm{CHF}$ receiver with four users at the spreading gain of $L=15$. User 1 is the desired user; interfering users arrive asynchronously with relative delays of 8,4 , and 2 chip intervals. All the users have equal power.

At a greater spreading factor, the advantage of chip-rate adaptation became evident. Fig. 5 summarizes the performance of the two types of receivers as a function of the spreading factor. All receivers are compared in the same configuration with $K=12, P=2, N=28$, and the feedback size $M=30$ chips for the CHF configuration, and $M=2,1$ or 0 symbols for the SDF configuration at $L=15,63$ and 255 , respectively. The $\mathrm{CHF}$ receiver gains about $3 \mathrm{~dB}$ over the SDF receiver at the spreading factor of 63 . Nonetheless, the SDF receiver provides very good performance in this case, given its lower complexity of implementation. At the spreading factor of 255, the difference in performance is striking: the CHF receiver gains about $10 \mathrm{~dB}$ over the SDF receiver, whose performance remains similar to that achieved with the lower spreading factors. While the CHF receiver demonstrates consistent performance improvement with increasing $L$, the SDF receiver does not. This observation is in agreement with the fact that symbolrate updating presents a trade-off between tracking accuracy and interference suppression capability. Simulation results that state to the same effect for a Rayleigh fading channel and a Doppler-shift-only channel are given in [10]. Although 255 is a high spreading factor to be used for supporting several users only, it is interesting to note the high quality of performance that can be obtained, and, thus, the margin left for the system if it needs to operate in higher interference or in the LPI regime.

It should be emphasized that the experimental data used to demonstrate the receiver algorithms were obtained in stationary conditions, with no spatial diversity between the users. 
Were there intentional mobility in the system, one could expect the SDF receiver to degrade more rapidly with an increase in spreading factor. We thus conclude that for the present experimental conditions, SDF receiver represents an excellent choice for a CDMA system operating with few users at a low spreading gain. When symbol-rate adaptation and feedback filtering are sufficient, this receiver offers minimal complexity of implementation. However, if a greater processing gain is desired (and the bandwidth expansion can be afforded) CHF receiver offers better performance, albeit at higher complexity.

Finally, it should be mentioned that reduction in computational requirements of the CHF receiver is possible through early discarding of some hypotheses. Namely, with a large spreading factor, there may be no need to wait for the entire code period before making a decision. Partial despreading can be performed instead, and those hypotheses that appear to be wrong, i.e. whose partial MSE fails to increase above a certain level, can be discarded, thus reducing the amount of computations per symbol.

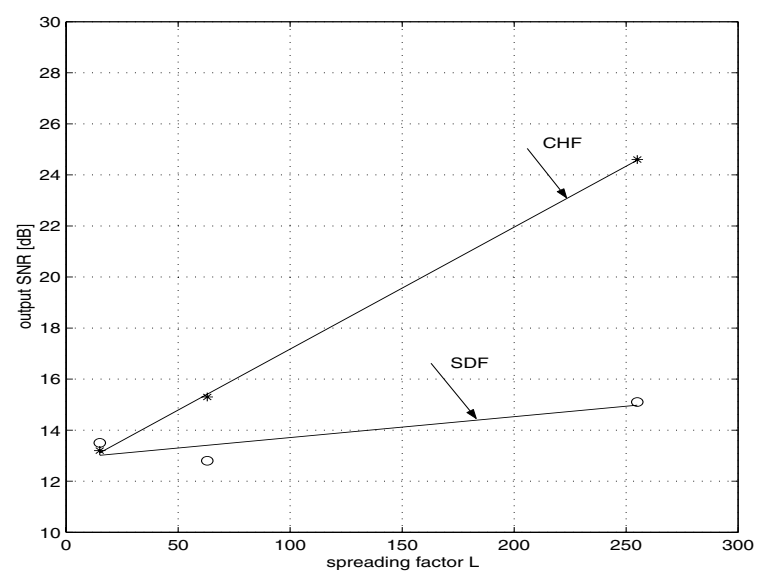

Fig. 5. Summary of experimental performance results: output SNR for the chip hypothesis feedback (CHF) and the symbol decision feedback (SDF) receivers as a function of the spreading factor. Four equal-power asynchronous users at present in the system. The adaptive algorithm used for the combiner/equalizer is RLS/LMS for the SDF receiver and LMS/LMS for the $\mathrm{CHF}$ receiver.

\section{CONCLUSIONS AND FURTHER WORK}

Direct-sequence CDMA is considered for use in future wideband mobile underwater acoustic networks, where a typical configuration may include several AUVs operating over a distance of a few kilometers to the central receiver, which can either be a fixed or a movable platform equipped with a receiving array for detection of asynchronous multiuser signals. For such a network, two types of multichannel detection algorithms were designed: the symbol decision feedback (SDF) receiver and the chip hypothesis feedback (CHF) receiver. The algorithms were tested using experimental data. Excellent performance was demonstrated, which owes to the integration of adaptive multichannel combining into the detection algorithms. Both of the receivers proposed offer a realistic platform for the next generation of acoustic modems that will support wideband acoustic CDMA communications.

The SDF receiver represents a minimal complexity solution and it is suitable for systems where the channel variation can be tracked at the symbol rate. In a wideband acoustic system, where full bandwidth utilization is accomplished by transmitting at maximal chip rate, the symbol rate is downscaled by the spreading factor. The spreading factor, and, consequently, the interference rejection capability, may thus be limited by the receiver's ability to track the channel at a symbol level. Channel variation is mostly influenced by the system mobility, and it can become a critical issue in a wideband AUV system. In such a situation, the CHF receiver should be used, as it performs fast chip-rate adaptation. At the price of increased computational complexity (but still only linear in the modulation level) this receiver provides improved performance, which consistently increases with the spreading factor used. While a large spreading factor may not be necessary in a small CDMA system, it is of interest to LPI systems. In the experimental study, using a chip rate of $19.2 \mathrm{kcps}$, it was found that the two receivers provided comparable performance at the spreading factor of 15 , while as the spreading factor increased to 63 and 255 , the $\mathrm{CHF}$ receiver gained advantage.

\section{ACKNOWLEDGMENT}

This work was supported by the Office of Naval Research, through the program "Demonstration of Undersea Autonomous Operation Capabilities and Related Technology Development."

\section{REFERENCES}

[1] E.M.Sozer, M.Stojanovic and J.G.Proakis, "Underwater acoustic networks," IEEE J. Oceanic Eng., vol.25, pp. 72-83, Jan. 2000.

[2] D.Kilfoyle and A.Baggeroer, "The state of the art in underwater acoustic telemetry," IEEE J. Oceanic Eng., vol.25, pp.4-27, Jan. 2000.

[3] M.Stojanovic, J.Catipovic and J.Proakis, "Reduced-complexity multichannel processing of underwater acoustic communication signals," J. Acoust. Soc. Am., 98(2), Pt.1, pp.961-972, Aug. 1995.

[4] M.Stojanovic and Z.Zvonar, "Multichannel processing of wideband multiuser communication signals in shallow water acoustic channels," IEEE J. Ocean. Eng., vol.21, Apr. 1996, pp.156-166.

[5] G.Woodward and B.Vucetic, "Adaptive detection for DS-CDMA," Proceedings of the IEEE, vol.86, No.7, pp.1413-1434, July 1998.

[6] M.Abdulrahman, A.Sheikh and D.Falconer, "Decision feedback equalization for CDMA indoor wireless communications," IEEE J. Select. Areas Commun., pp.698-706, May 1994.

[7] J.E.Smee and S.C.Schwartz, "Adaptive space-time feedforward/feedback detection for high data rate CDMA in frequencyselective fading," IEEE Trans. Commun., vol.49, No.2, pp.317-328, Feb. 2001.

[8] J.F.Rossler, L.H.-J.Lampe, W.H.Gerstacker and W.H.Huber, "Decision-feedback equalization for CDMA downlink," in Proc. Vehicular Technology Conference (VTC) 2002, vol.2, pp.816 - 820, May 2002.

[9] A.Santoso, J.Choi and B.Vucetic, "Chip level decision feedback equalizer for CDMA downlink with space-time coding," IEEE Electronic Letters, vol.7, No.7, pp.437-439, April 2004.

[10] M.Stojanovic and L.Freitag, "Hypothesis-feedback equalization for direct-sequence spread spectrum underwater communications," in Proc. IEEE Oceans'00 Conference, Sept. 2000.

[11] M.Stojanovic and L.Freitag, "Multichannel detection for wideband underwater acoustic CDMA communications," submitted to IEEE J. Oceanic Eng., Dec. 2004. Available upon request through e-mail. 